\title{
Influence of doping on the spin dynamics and magnetoelectric effect in hexagonal $\mathrm{Y}_{0.7} \mathrm{Lu}_{0.3} \mathrm{MnO}_{3}$
}

\author{
W. Tian, ${ }^{1, *}$ Guotai Tan, ${ }^{2,3, \dagger}$ Liu Liu, ${ }^{2}$ Jinxing Zhang, ${ }^{2}$ Barry Winn, ${ }^{1}$ Tao Hong, ${ }^{1}$ J. A. Fernandez-Baca, ${ }^{1,3}$ Chenglin Zhang, ${ }^{3,4}$ \\ and Pengcheng Dai ${ }^{3,4}$ \\ ${ }^{1}$ Quantum Condensed Matter Division, Oak Ridge National Laboratory, Oak Ridge, Tennessee 37831, USA \\ ${ }^{2}$ Department of Physics, Beijing Normal University, Beijing 100875, China \\ ${ }^{3}$ Department of Physics and Astronomy, The University of Tennessee, Knoxville, Tennessee 37996, USA \\ ${ }^{4}$ Department of Physics and Astronomy, Rice University, Houston, Texas 77005, USA \\ (Received 9 August 2013; revised manuscript received 3 April 2014; published 18 April 2014)
}

\begin{abstract}
We use inelastic neutron scattering and dielectric constant measurements to study the doping influence on the spin dynamics and magnetoelectric (ME) effect in hexagonal $\mathrm{Y}_{0.7} \mathrm{Lu}_{0.3} \mathrm{MnO}_{3}$. In undoped $\mathrm{YMnO}_{3}$ and $\mathrm{LuMnO}_{3}$, the $\mathrm{Mn}$ trimerization distortion has been suggested to play a key role in determining the magnetic structure and the magnetoelectric effect. In $\mathrm{Y}_{0.7} \mathrm{Lu}_{0.3} \mathrm{MnO}_{3}$, at the antiferromagnetic zone center, we observed a much smaller $\Delta_{12} \approx 0.52 \mathrm{meV}$ gap (which is $\sim 2.5 \mathrm{meV}$ for both $\mathrm{YMnO}_{3}$ and $\mathrm{LuMnO}_{3}$ ) that coincides with a weaker in-plane dielectric anomaly at $T_{N}$; both can be attributed to a weaker Mn trimerization distortion in $\mathrm{Y}_{0.7} \mathrm{Lu}_{0.3} \mathrm{MnO}_{3}$ compared to $\mathrm{YMnO}_{3}$ and $\mathrm{LuMnO}_{3}$. The results provide strong evidence that the magnitude of $\mathrm{ME}$ coupling is linked to the strength of the trimerization distortion, suggesting the Mn trimerization is responsible for the ME effect in $\mathrm{Y}_{1-y} \mathrm{Lu}_{y} \mathrm{MnO}_{3}$.
\end{abstract}

DOI: 10.1103/PhysRevB.89.144417

PACS number(s): 75.85.+t, 75.30.Ds, 75.40.Gb, 75.50.Ee

Driven by modern technology trends towards device miniaturization, there is considerable interest in multiferroic materials, which exhibit both magnetic order and electrical polarization [1-7]. The hexagonal manganite $R \mathrm{MnO}_{3}[8,9]$ (where $R$ is a rare-earth element with relatively small ionic radius) is a prototypical example of the so-called type-I multiferroics [10] with ferroelectric order at $T_{c} \sim 900 \mathrm{~K}$ [11] and an antiferromagnetic (AFM) order at much lower temperature, $T_{N} \sim 100 \mathrm{~K}$ [12]. A large dielectric anomaly occurs at $T_{N}$ [13-15] indicating strong magnetoelectric (ME) coupling in these materials. There has been a large amount of experimental work in recent years, with the aim to understand the microscopic mechanism for the coupling between magnetic and electric degrees of freedom in these materials. Although it is generally believed that the spin-lattice coupling plays an important role in determining the complex properties in $R \mathrm{MnO}_{3}$, much is unclear concerning the factors that influence the magnitude of the ME coupling [16,17]. In the present paper, we use inelastic neutron scattering (INS) and dielectric constant measurements to show that the magnitude of the ME coupling is directly coupled to the strength of the Mn trimerization distortion in $\mathrm{Y}_{0.7} \mathrm{Lu}_{0.3} \mathrm{MnO}_{3}$ in the AFM phase. Our results thus provide strong evidence that the $\mathrm{Mn}$ trimerization is responsible for the ME effect and multiferroic phenomenon in $\mathrm{Y}_{1-y} \mathrm{Lu}_{y} \mathrm{MnO}_{3}$.

Undoped $\mathrm{YMnO}_{3}$ and $\mathrm{LuMnO}_{3}$ are characteristic hexagonal manganites with the $\mathrm{Mn}^{3+}$ ions at the $x \sim 1 / 3$ position forming a nearly ideal triangular lattice in the $a b$ plane above $T_{N} . \mathrm{YMnO}_{3}$ and $\mathrm{LuMnO}_{3}$ undergo AFM transitions (to two different magnetic structures) at $T_{N} \sim 75 \mathrm{~K}$ and $T_{N} \sim 88 \mathrm{~K}$ respectively, accompanied by an isostructural transition with large atomic displacement for all atoms in the unit cell. In particular, a distinct change of the $\mathrm{Mn}$ atomic position, namely the Mn trimerization distortion, occurs in the basal plane at $T_{N}$

\footnotetext{
*wt6@ornl.gov

†tanbj2008@gmail.com
}

[17]. As illustrated in Fig. 1, the Mn trimers distort in opposite directions in $\mathrm{YMnO}_{3}$ and $\mathrm{LuMnO}_{3}$, expanding for $\mathrm{YMnO}_{3}$ and contracting for $\mathrm{LuMnO}_{3}$. A recent theoretical study finds that the different magnetic structures of $\mathrm{YMnO}_{3}$ and $\mathrm{LuMnO}_{3}$ are determined by the different trimerization directions in these compounds [18]. Moreover, the dielectric anomaly at $T_{N}$ is observed only in $\varepsilon_{a b}$ but not in $\varepsilon_{c}$ for both $\mathrm{YMnO}_{3}$ and $\mathrm{LuMnO}_{3}$ [15]. Although these studies suggest that the Mn trimerization may play a key role in determining the magnetic structure and the ME effects in $\mathrm{Y}_{1-y} \mathrm{Lu}_{y} \mathrm{MnO}_{3}$, there are no experimental studies to determine the connection between the $\mathrm{Mn}$ trimerization and $\mathrm{ME}$ coupling. $\mathrm{Y}_{1-y} \mathrm{Lu}_{y} \mathrm{MnO}_{3}$ is an ideal system for such a study due to the following reasons: (1) since both $\mathrm{Y}$ and $\mathrm{Lu}$ are nonmagnetic, $\mathrm{Y}_{1-y} \mathrm{Lu}_{y} \mathrm{MnO}_{3}$ is a clean system to study the magnetism of the Mn triangular lattice and its correlation with the ME effects; (2) the strength of the $\mathrm{Mn}$ trimerization distortion can be tuned in $\mathrm{Y}_{1-y} \mathrm{Lu}_{y} \mathrm{MnO}_{3}$. At $10 \mathrm{~K}$, with increasing Lu concentration $y$, the $\mathrm{Mn}$ atomic position $x$ changes from 0.340 for $\mathrm{YMnO}_{3}$, larger than $x_{c}=1 / 3$ for an ideal triangular lattice, to 0.331 for $\mathrm{LuMnO}_{3}$, smaller than $1 / 3$. At $y \sim 0.3$, the $\mathrm{Mn}$ atomic position $x$ is very close to the critical value $x_{c}=1 / 3$; we thus expect a much weaker trimerization distortion in $\mathrm{Y}_{0.7} \mathrm{Lu}_{0.3} \mathrm{MnO}_{3}$ [19].

All measurements reported here were performed on single-crystal samples. Large $\mathrm{Y}_{1-y} \mathrm{Lu}_{y} \mathrm{MnO}_{3}$ single crystals with nominal value of $y=0.3$ were grown by the floating zone method under 4 atmospheres of oxygen flow. The crystals cut from the long rods were then annealed at $1350{ }^{\circ} \mathrm{C}$ for 24 hours in a flowing argon atmosphere. For the magnetic susceptibility and dielectric constant measurements, the single crystal was cut into thin plates with $a b$ axes lying in the plane and the $c$ axis pointing out of the plane. The magnetic susceptibility was measured using a Quantum Design magnetic properties measurement system with magnetic field applied along the $c$ axis. The dielectric constant was measured using an inductance-capacitance-resistance (LCR) meter with electric field applied perpendicular and parallel to the $c$ axis, and data were taken at $3.5 \mathrm{~V}$ ac driving voltage and $100 \mathrm{kHz}$ frequency. 


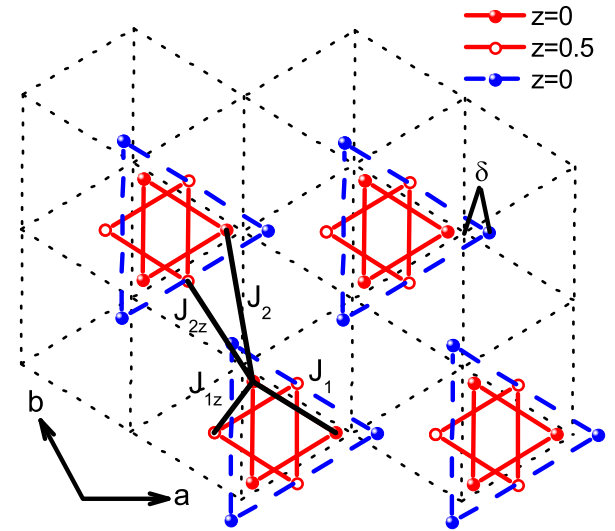

FIG. 1. (Color online) Schematic drawing of the Mn trimerization distortion below $T_{N}$. The dotted line depicts the ideal triangular lattice in the hexagonal $a b$ plane with $\mathrm{Mn}$ ions at the $x_{c}=1 / 3$ position. The solid blue and red circles represent $\mathrm{Mn}$ ions in the $z=0$ plane, illustrating the opposite distortion directions of the $\mathrm{Mn}$ trimers: expansion in $\mathrm{YMnO}_{3}$ (blue) and contraction in $\mathrm{LuMnO}_{3}$ (red). For $\mathrm{LuMnO}_{3}$, the $\mathrm{Mn}$ ions in the $z=0.5$ plane are shown as red open circles, and the in-plane $\left(J_{1}, J_{2}\right)$ and interplane $\left(J_{1 z}, J_{2 z}\right)$ magnetic exchange interactions are shown by solid lines. $\delta=\left|x-x_{c}\right|$ is the trimerization distortion parameter as described in the text.

A single crystal with a mass of $\sim 4$ grams was used for the neutron scattering experiments. The crystal was mounted on a aluminum plate and oriented in the $\left(\begin{array}{lll}H & 0 & L\end{array}\right)$ scattering plane. The sample was then sealed in aluminum sample can under helium atmosphere and cooled using a closed-cycle $\mathrm{He}$ refrigerator. The neutron experiments were carried out using the HB-1A and CTAX triple-axis spectrometers (TAS) located at the High Flux Isotope Reactor (HFIR), and the Hybrid Spectrometer (HYSPEC) located at the Spallation Neutron Source (SNS) at Oak Ridge National Laboratory. HB-1A is a fixed incident energy TAS $\left(E_{i}=14.64 \mathrm{meV}\right)$, and CTAX is a cold neutron TAS. Collimations of $48^{\prime}-48^{\prime}$-sample- $40^{\prime}-80^{\prime}$ downstream from the reactor to the detector was used for the HB-1A experiment with two pyrolitic graphite (PG) filters placed before the sample to eliminate higher-order contaminations in the beam. The CTAX experiment was performed with a fixed final energy of $E_{f}=3 \mathrm{meV}$ (energy resolution is $\sim 0.1 \mathrm{meV}$ FWHM at elastic condition) and collimations of guide-open-sample- $80^{\prime}$-open. Higher-order contaminations were removed by a cooled Be filter placed between the sample and the analyzer. The HYSPEC experiment was carried out using an incident energy of $E_{i}=25 \mathrm{meV}$ with a Fermi chopper spinning at $420 \mathrm{~Hz}$.

The $\mathrm{Y}_{0.7} \mathrm{Lu}_{0.3} \mathrm{MnO}_{3}$ crystal was characterized by the magnetic susceptibility and neutron scattering magnetic order parameter measurements. Figure 2(a) shows the magnetic susceptibility measured with $H \| c$ exhibiting a kink at $\sim 78 \mathrm{~K}$ indicating the AFM transition. The order parameter plotted in Fig. 2(b) was measured by monitoring the strong magnetic Bragg peak (1 0101$)$ as a function of temperature. The integrated intensity was obtained by fitting the $\left(\begin{array}{lll}1 & 0 & 1\end{array}\right)$ rocking curve measured at each temperature to a Gaussian function with a constant background. As illustrated by the dashed green line, the AFM transition at $T_{N} \sim 78 \mathrm{~K}$ was observed in

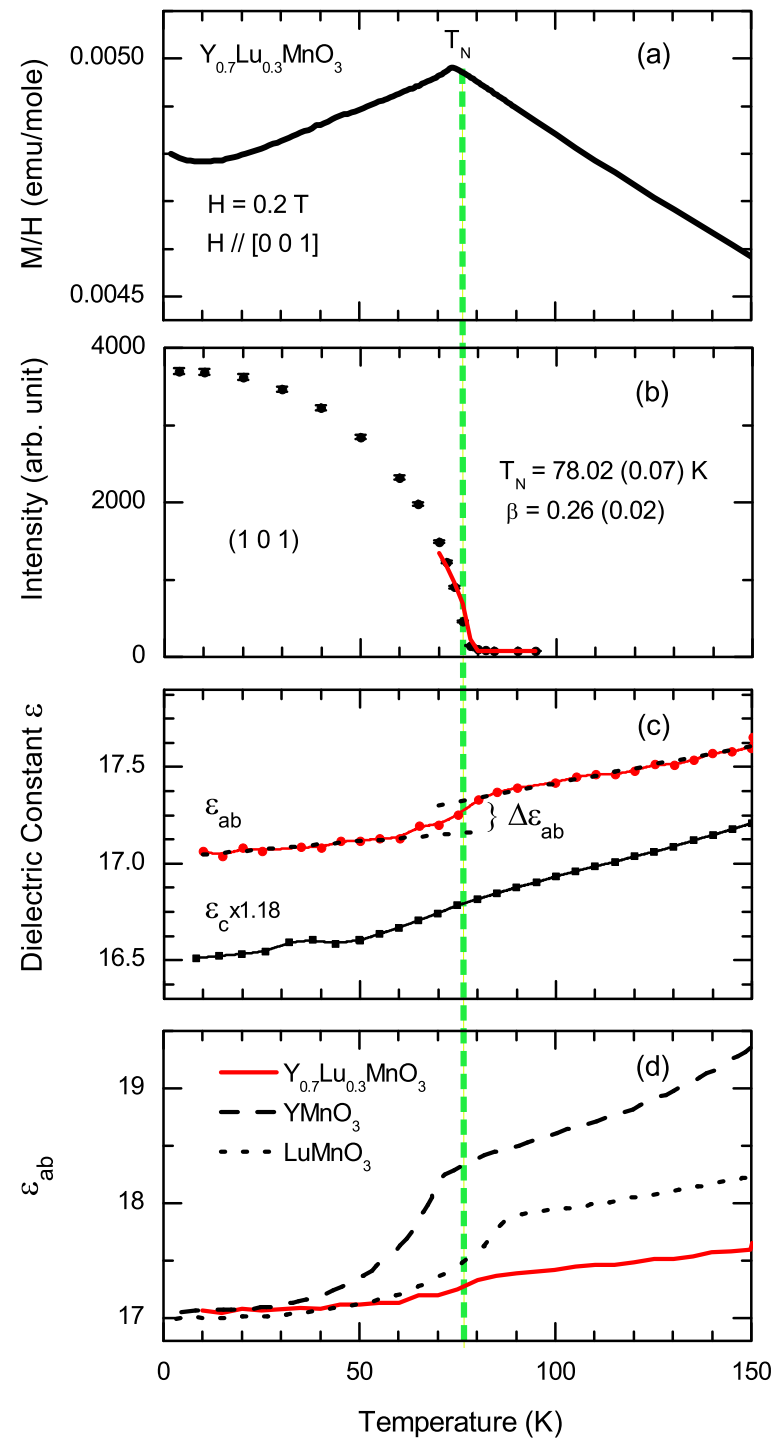

FIG. 2. (Color online) Magnetic susceptibility, magnetic order parameter, and dielectric constant measurements of $\mathrm{Y}_{0.7} \mathrm{Lu}_{0.3} \mathrm{MnO}_{3}$. The dashed green line depicts the Neel temperature $T_{N}$. (a) Magnetic susceptibility versus temperature measured with applied magnetic field parallel to the $c$ axis. (b) Integrated intensity of the $\left(\begin{array}{lll}1 & 0 & 1\end{array}\right)$ magnetic Bragg reflection as a function of temperature. The solid red line is a fit of the data to the power law as described in the text. (c) In-plane and out-of-plane dielectric constant measured with the electric field applied perpendicular and parallel to the $c$ axis. $\Delta \varepsilon_{a b}$ defines the critical in-plane dielectric constant change at $T_{N}$. (d) Comparison of the in-plane dielectric constant $\varepsilon_{a b}$ between $\mathrm{YMnO}_{3}, \mathrm{LuMnO}_{3}$, and $\mathrm{Y}_{0.7} \mathrm{Lu}_{0.3} \mathrm{MnO}_{3}$. The data for $\mathrm{YMnO}_{3}\left(\varepsilon_{a b}-\right.$ 2.1) and $\mathrm{LuMnO}_{3}\left(\varepsilon_{a b}+0.3\right)$ are from Ref. [15] and are plotted with offsets of -2.1 and 0.3 for $\mathrm{YMnO}_{3}$ and $\mathrm{LuMnO}_{3}$, respectively.

both measurements, consistent with previous reports [19]. The solid red line in Fig. 2 (b) is a fit to a power-law $\left.I(T)=I_{0}\left[\left(T_{N}-T\right) / T_{N}\right)\right]^{2 \beta}$ that yields $T_{N} \approx 78.02 \pm 0.07$ $\mathrm{K}$ and $\beta \approx 0.26 \pm 0.02$, where $\beta$ is the critical exponent. The yielded $\beta \sim 0.26$ value is between the theoretical values of a two-dimensional $(\beta=0.125)$ and a three-dimensional ( $\beta=0.326)$ Ising systems, in good agreement with a prior study [20]. 


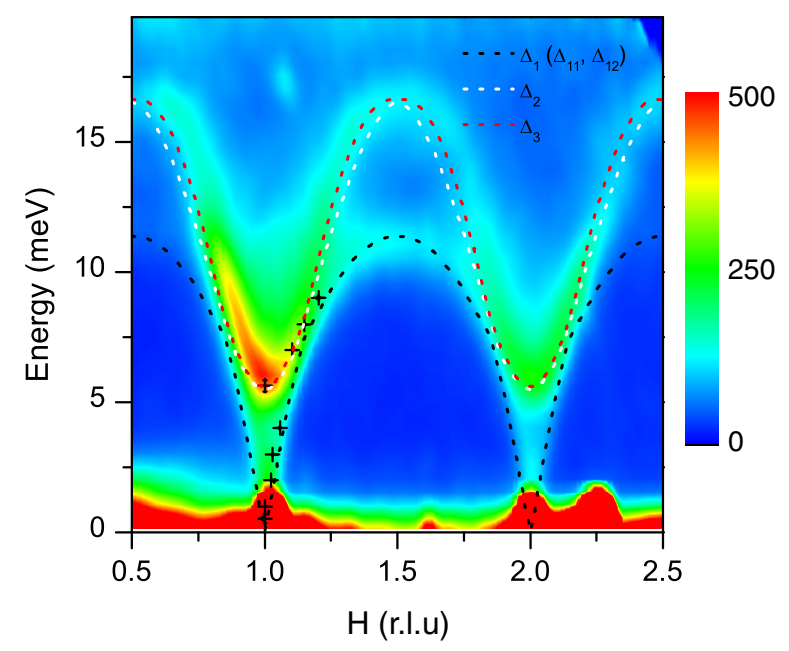

FIG. 3. (Color online) INS spectra of $\mathrm{Y}_{0.7} \mathrm{Lu}_{0.3} \mathrm{MnO}_{3}$ along $(H$ 0 0) from the HYSPEC measurements at $4 \mathrm{~K}$. The crossed symbols are data points obtained from TAS measurements. The dotted lines are the calculated dispersion (six branches appear as three doubly degenerate branches) from the spin-wave model as described in the text.

The spin dynamics of $\mathrm{Mn}^{3+}$ ions has been investigated in detail in $\mathrm{YMnO}_{3}, \mathrm{LuMnO}_{3}$, and $\mathrm{HoMnO}_{3}$ [20-24]. There are six $\mathrm{Mn}^{3+}$ ions in the magnetic unit cell that lead to six magnon branches in these materials. However, due to the fact that the interplane magnetic coupling is much weaker compared to the in-plane magnetic exchange interaction, the spin-wave dispersion in the hexagonal $a b$ plane appears as three branches $\left(\Delta_{1}, \Delta_{2}\right.$, and $\Delta_{3}$ as depicted in Fig. 3), each containing two nearly doubly degenerate modes $[23,24]$. Only the degeneracy of the $\Delta_{1}$ low energy branch can be lifted at the zone center [becoming $\Delta_{11}$ and $\Delta_{12}$ as shown in Figs. 4(a) and 4(b)] and along $L$ due to interlayer coupling. Figure 3 shows the INS spectra of $\mathrm{Y}_{0.7} \mathrm{Lu}_{0.3} \mathrm{MnO}_{3}$ projected along the $\left(\begin{array}{lll}H & 0 & 0\end{array}\right)$ direction, where the crossed symbols are data points from TAS measurements, in good agreement with the HYSPEC data. Figure 4 summarizes the TAS data measured along $L$. As shown in Figs. 4(a)-4(c), at the magnetic zone center, two modes were observed in $\mathrm{Y}_{0.7} \mathrm{Lu}_{0.3} \mathrm{MnO}_{3}, \Delta_{12} \approx 0.52 \mathrm{meV}\left[\right.$ Fig. 4(b)] and $\Delta_{3}\left(\approx \Delta_{2}\right) \approx$ $5.56 \mathrm{meV}$ [Fig. 4 (c)], whereas the lowest energy mode $\Delta_{11} \approx 0 \mathrm{meV}$ [Fig. 4 (a)] is gapless within instrumental resolution ( $\sim 0.1 \mathrm{meV}$ FWHM at elastic condition). In comparison, previous study has shown that $\Delta_{11} \approx 0.22 \mathrm{meV}$, $\Delta_{12} \approx 2.4 \mathrm{meV}$, and $\Delta_{3} \approx 5.4 \mathrm{meV}$ in $\mathrm{YMnO}_{3}$ [20,23]; and $\Delta_{11} \approx 0 \mathrm{meV}, \Delta_{12} \approx 2.5 \mathrm{meV}$, and $\Delta_{3} \approx 6.5 \mathrm{meV}$ in $\mathrm{LuMnO}_{3}$ [24]. Figure 4(d) plots the derived dispersion curves along $L$ by fitting the energy scans at constant wave vector, assuming Gaussian peak shape. Similar to that reported for $\mathrm{YMnO}_{3}$ and $\mathrm{LuMnO}_{3}$, strong dispersions were observed along $H$ (Fig. 3) with an overall bandwidth of $\sim 17 \mathrm{meV}$ (which is $\sim 16$ and $21 \mathrm{meV}$ for $\mathrm{YMnO}_{3}$ and $\mathrm{LuMnO}_{3}$ ), and weak dispersions were observed along $L$ [Fig. 4 (d)] consistent with the layered magnetic structure of $\mathrm{Y}_{1-y} \mathrm{Lu}_{y} \mathrm{MnO}_{3}$. The $\sim 5.5 \mathrm{meV}$ mode (containing four degenerate modes from upper $\Delta_{2}$ and $\Delta_{3}$ branches) is dispersionless along $L$ within the instrumental resolution. Overall, the $q$ dependence of the magnetic spectra of $\mathrm{Y}_{0.7} \mathrm{Lu}_{0.3} \mathrm{MnO}_{3}$ shows very similar behavior compared to $\mathrm{YMnO}_{3}$ and $\mathrm{LuMnO}_{3}$, except that unlike $\mathrm{YMnO}_{3}$ and $\mathrm{LuMnO}_{3}$ which have almost the same value of $\Delta_{12} \approx 2.5 \mathrm{meV}$ regardless of the opposite trimerization distortion direction in these materials, a much smaller $\Delta_{12} \approx 0.52 \mathrm{meV}$ was observed in $\mathrm{Y}_{0.7} \mathrm{Lu}_{0.3} \mathrm{MnO}_{3}$.

In order to make a quantitative comparison between $\mathrm{YMnO}_{3}, \mathrm{Y}_{0.7} \mathrm{Lu}_{0.3} \mathrm{MnO}_{3}$, and $\mathrm{LuMnO}_{3}$, we analyze the observed $\mathrm{Y}_{0.7} \mathrm{Lu}_{0.3} \mathrm{MnO}_{3}$ spin-wave dispersion using the same model that has been applied to $\mathrm{YMnO}_{3}$ in Ref. [23] and $\mathrm{LuMnO}_{3}$ in Ref. [24]. The spin Hamiltonian can be described by the following equation:

$$
\mathcal{H}=-\sum_{\langle i j\rangle} J_{i j} \vec{S}_{i} \cdot \vec{S}_{j}-D_{1} \sum_{i}\left(S_{i}^{z}\right)^{2}-D_{2} \sum_{i}\left(\vec{S}_{i} \cdot \vec{n}_{i}\right)^{2}
$$

which takes into account two in-plane $\left(J_{1}, J_{2}\right)$ and two interplane $\left(J_{1 z}, J_{2 z}\right)$ exchange interactions as depicted in Fig. 1, and $D_{1}$ and $D_{2}$ represent the out-of-plane and in-plane anisotropies. The direction of the in-plane anisotropy $D_{2}$ is parallel to the spin directions, $\vec{n}_{i}=\left\langle\vec{S}_{i}\right\rangle /\left|\left\langle\vec{S}_{i}\right\rangle\right|$. Using the program implemented in the MCPHASE software package [25], a fit to the $\mathrm{Y}_{0.7} \mathrm{Lu}_{0.3} \mathrm{MnO}_{3}$ data yields $J_{1}=-2.65(5)$ $\mathrm{meV}, J_{2}=-2.32(5) \mathrm{meV}, J_{2 z}=0.0012$ (4) $\mathrm{meV}$, and $D_{1}=$ $-0.44(1) \mathrm{meV}$ under the following constraints: (1) Our neutron diffraction measurements show that $\mathrm{Y}_{0.7} \mathrm{Lu}_{0.3} \mathrm{MnO}_{3}$ adopted the same magnetic structure as $\mathrm{LuMnO}_{3}$, thus the same spin-wave model used for $\mathrm{LuMnO}_{3}$ [24] in the MCPHASE package can be applied to analyze the $\mathrm{Y}_{0.7} \mathrm{Lu}_{0.3} \mathrm{MnO}_{3}$ data. (2) The in-plane anisotropy $D_{2}$ term is needed in $\mathrm{YMnO}_{3}$ because of the $\Delta_{11} \approx 0.22 \mathrm{meV}$ gap, but it can be set to $D_{2}=0$ in $\mathrm{Y}_{0.7} \mathrm{Lu}_{0.3} \mathrm{MnO}_{3}$ and $\mathrm{LuMnO}_{3}$ because the $\Delta_{11}$ gap is too small to be detected. (3) As shown in Figs. 3 and 4(d), the degeneracy of the $\Delta_{1}$ mode is lifted and becomes $\Delta_{11}$ and $\Delta_{12}$ at the zone center and along $L$ due to the interplane coupling [23,24], whereas the upper $\Delta_{2} \approx \Delta_{3}$ mode remains degenerate, which precludes $J_{1 z}$ and $J_{2 z}$ being determined independently [23,24]; therefore $J_{1 z}=0$ is fixed and we obtain the difference of $\left|J_{1 z}-J_{2 z}\right|$ from the fitting. As illustrated in Fig. 3 comparing the calculated results to the measured magnetic spectra, our INS data can be well described by this model.

In Table I we list the fitted parameters for $\mathrm{Y}_{0.7} \mathrm{Lu}_{0.3} \mathrm{MnO}_{3}$ in comparison with those for $\mathrm{YMnO}_{3}$ and $\mathrm{LuMnO}_{3}$. It shows that the lattice constants, $T_{N}$, and $D_{1}$ values of $\mathrm{Y}_{0.7} \mathrm{Lu}_{0.3} \mathrm{MnO}_{3}$ fall in between the values of $\mathrm{YMnO}_{3}$ and $\mathrm{LuMnO}_{3}$, consistent with the fact that both the lattice parameters and the unit cell volume are contracted from $\mathrm{YMnO}_{3}$ to $\mathrm{LuMnO}_{3}$. On the other hand, $J_{1} \approx J_{2}$ is obtained, and in particular a significantly smaller $\left|J_{1 z}-J_{2 z}\right| \approx 0.0012(\mathrm{meV})$, at least 10 times smaller compared to $\mathrm{YMnO}_{3}$ and $\mathrm{LuMnO}_{3}$, is obtained in $\mathrm{Y}_{0.7} \mathrm{Lu}_{0.3} \mathrm{MnO}_{3}$. We will discuss below that the smaller $\Delta_{12} \approx 0.52 \mathrm{meV}$ gap observed in $\mathrm{Y}_{0.7} \mathrm{Lu}_{0.3} \mathrm{MnO}_{3}$ is associated with the weaker Mn trimerization distortion in this material.

The analytic expressions of the energy gaps at the magnetic zone center have been given in a previous study [23] for the spin Hamiltonian [Eq. (1)] assuming very weak interplane 

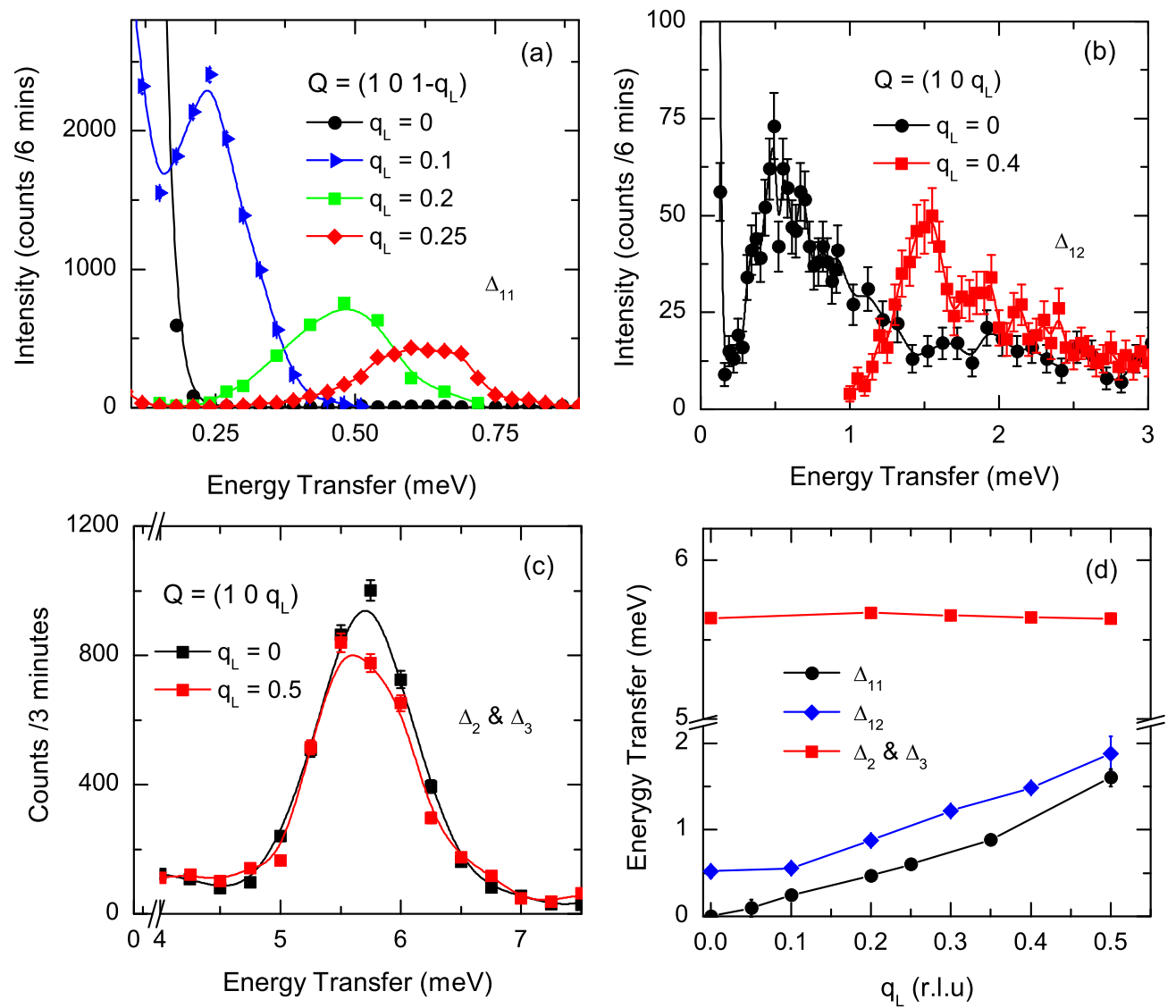

FIG. 4. (Color online) TAS data measured at $4 \mathrm{~K}$ illustrates the $q$ dependence of the magnetic excitations along $L$ in $\mathrm{Y}_{0.7} \mathrm{Lu}_{0.3} \mathrm{MnO}_{3}$. (a) The $\Delta_{11}$ gapless mode measured at selected $\left(\begin{array}{lll}1 & 0 & 1-q_{L}\end{array}\right)$ and (b) the $\Delta_{12} \approx 0.52 \mathrm{meV}$ mode measured at selected ( $\left.10 q_{L}\right)$ using the CG-4C cold neutron TAS. (c) The $\Delta_{2} \approx \Delta_{3} \approx 5.56 \mathrm{meV}$ mode measured at selected ( $\left.10 q_{L}\right)$ using the HB-1A thermal neutron TAS. (d) Spin-wave dispersion along the $L$ direction constructed from a series of energy scans at constant wave vector.

couplings $J_{1 z}$ and $J_{2 z}$, and in-plane anisotropy $D_{2}$ :

$\Delta_{11} \simeq 2 S \sqrt{-D_{2} \lambda_{1}}$

$\Delta_{12} \simeq 2 S \sqrt{-D_{2} \lambda_{1}-2\left(J_{1 z}-J_{2 z}\right) \lambda_{1}}$,

$\Delta_{2} \simeq S \sqrt{2\left(D_{1} \lambda_{2}-D_{2} \lambda_{3}-2 D_{1} J_{1 z}\right)}$,

$\Delta_{3} \simeq S \sqrt{2\left[D_{1} \lambda_{2}-D_{2} \lambda_{3}-D_{1}\left(J_{1 z}-4 J_{2 z}\right)-2\left(J_{1 z}-J_{2 z}\right) \lambda_{2}\right]}$,

where $\lambda_{1}, \lambda_{2}$ and $\lambda_{3}$ are defined as $\lambda_{1}=D_{1}+(3 / 2) J_{1}+3 J_{2}$, $\lambda_{2}=(3 / 2) J_{1}+3 J_{2}$, and $\lambda_{3}=2 D_{1}+(3 / 2) J_{1}+3 J_{2}$, respec- tively. As indicated in the above equation, the $\Delta_{12}$ gap is determined by $\lambda_{1}, D_{2}$, and $J_{1 z}-J_{2 z}$. Based on the obtained fitting parameters listed in Table I, $\lambda_{1}$ is almost the same between the three compounds: $\lambda_{1}=-11.44,-11.375$, and $-11.235 \mathrm{meV}$ for $\mathrm{YMnO}_{3}, \mathrm{Y}_{0.7} \mathrm{Lu}_{0.3} \mathrm{MnO}_{3}$, and $\mathrm{LuMnO}_{3}$, respectively. Due to $D_{2} \approx 0.0007 \mathrm{meV}$ being very small for $\mathrm{YMnO}_{3}$, and $D_{2}=0$ being fixed for $\mathrm{Y}_{0.7} \mathrm{Lu}_{0.3} \mathrm{MnO}_{3}$ and $\mathrm{LuMnO}_{3}$, the $D_{2} \lambda_{1}$ term in the $\Delta_{12}$ equation can be neglected $\left(D_{2} \lambda_{1}=0\right.$ for $\mathrm{Y}_{0.7} \mathrm{Lu}_{0.3} \mathrm{MnO}_{3}$ and $\mathrm{LuMnO}_{3}$, and $D_{2} \lambda_{1}=0.008$ for $\mathrm{YMnO}_{3}$ ). Therefore the $\Delta_{12} \approx 0.52 \mathrm{meV}$

TABLE I. Comparison of the lattice constants (space group $P 6_{3} \mathrm{~cm}$ ), $T_{N}$, in-plane exchange constants $J_{1}$ and $J_{2}$, difference between interplane exchange couplings $\left|J_{1 z}-J_{2 z}\right|$, out-of-plane and in-plane anisotropy parameters $D_{1}$ and $D_{2}$, trimerization distortion parameter $\delta$, and the critical dielectric constant change parameter $\Delta \varepsilon_{a b}$ between $\mathrm{YMnO}_{3}, \mathrm{LuMnO}_{3}$, and $\mathrm{Y}_{0.7} \mathrm{Lu}_{0.3} \mathrm{MnO}_{3}$.

\begin{tabular}{lccc}
\hline \hline & $\mathrm{YMnO}_{3}($ Ref. [23]) & $\mathrm{Y}_{0.7} \mathrm{Lu}_{0.3} \mathrm{MnO}_{3}$ (this work) & $\mathrm{LuMnO}_{3}(\mathrm{Ref} .[24])$ \\
\hline Lattice $(\AA)$ & $a=6.132, c=11.452$ & $a=6.103(2), c=11.403(1)$ & $a=6.05, c=11.4$ \\
$T_{N}(\mathrm{~K})$ & 75 & 78 & 88 \\
$J_{1}(\mathrm{meV})$ & $-3.4(2)$ & $-2.65(5)$ & $-4.09(2)$ \\
$J_{2}(\mathrm{meV})$ & $-2.02(7)$ & $-2.32(5)$ & $-1.54(5)$ \\
$\left|J_{1 z}-J_{2 z}\right|(\mathrm{meV})$ & $0.014(2)$ & $0.0012(4)$ & $0.019(2)$ \\
$D_{1}(\mathrm{meV})$ & $-0.28(1)$ & $-0.44(1)$ & $-0.48(1)$ \\
$D_{2}(\mathrm{meV})$ & $0.0007(6)$ & 0 & 0 \\
$\delta$ & 0.007 & 0.001 & 0.003 \\
$\Delta \varepsilon_{a b}$ & 1.02 & 0.17 & 0.77 \\
\hline \hline
\end{tabular}


gap observed in $\mathrm{Y}_{0.7} \mathrm{Lu}_{0.3} \mathrm{MnO}_{3}$ is due to a much smaller $\left|J_{1 z}-J_{2 z}\right|$. The differences between both in-plane and interplane exchange constants $J_{1}-J_{2}$ and $J_{1 z}-J_{2 z}$ can serve as good parameters that are very sensitive to the strength of the trimerization distortion, regardless of the small differences in lattice parameters. Smaller $J_{1}-J_{2}$ and $J_{1 z}-J_{2 z}$ values correspond to weaker trimerization distortion, with the extreme case of $J_{1}-J_{2}=0$ and $J_{1 z}-J_{2 z}=0$ for an ideal triangular lattice. The obtained $J_{1}-J_{2} \approx 0.33 \mathrm{meV}$ and $J_{1 z}-J_{2 z} \approx 0.0012 \mathrm{meV}$ both indicate a much weaker $\mathrm{Mn}$ trimerization distortion in $\mathrm{Y}_{0.7} \mathrm{Lu}_{0.3} \mathrm{MnO}_{3}$, consistent with a previous systematic study showing that the $\mathrm{Mn}$ atomic position in $\mathrm{Y}_{0.7} \mathrm{Lu}_{0.3} \mathrm{MnO}_{3}$ is very close to the critical value $x \sim 1 / 3$ [19].

Our spin-wave study indicates that the smaller $\Delta_{12}$ gap observed in $\mathrm{Y}_{0.7} \mathrm{Lu}_{0.3} \mathrm{MnO}_{3}$ is associated with the weaker $\mathrm{Mn}$ trimerization distortion in this material. A dielectric anomaly was observed in both $\mathrm{YMnO}_{3}$ and $\mathrm{LuMnO}_{3}$; it is thus of great interest to see how the weaker Mn trimerization distortion affects the magnitude of the ME coupling. If the ME effect is directly coupled to the Mn trimerization distortion, we would expect a much weaker in-plane dielectric anomaly in $\mathrm{Y}_{0.7} \mathrm{Lu}_{0.3} \mathrm{MnO}_{3}$, which is indeed what we observed in the dielectric constant measurements. As illustrated in Fig. 2(c), at $T_{N}$ no anomaly was observed in $\varepsilon_{c}$, consistent with previous reports, whereas a weaker dielectric anomaly was observed in $\varepsilon_{a b}$. Figure 2(d) compares the in-plane dielectric constant $\varepsilon_{a b}$ between $\mathrm{YMnO}_{3}, \mathrm{LuMnO}_{3}$, and $\mathrm{Y}_{0.7} \mathrm{Lu}_{0.3} \mathrm{MnO}_{3}\left(\varepsilon_{a b}\right.$ values for $\mathrm{YMnO}_{3}$ and $\mathrm{LuMnO}_{3}$ are taken from Ref. [15] and plotted in Fig. 2(d) with -2.1 and 0.3 offsets, respectively) and it clearly shows that the dielectric anomaly in $\mathrm{Y}_{0.7} \mathrm{Lu}_{0.3} \mathrm{MnO}_{3}$ is much weaker compared to $\mathrm{YMnO}_{3}$ and $\mathrm{LuMnO}_{3}$.

To illustrate how the strength of the trimerization distortion affects spin dynamics $\left(\Delta_{12}\right.$ gap) and the in-plane dielectric anomaly, we define a trimerization distortion parameter $\delta=\left|x-x_{c}\right|$ as depicted in Fig. 1 to reflect the strength of the trimerization distortion. We also define a critical dielectric constant change parameter $\Delta \varepsilon_{a b}$ to represent the magnitude of the ME coupling at $T_{N}$. As shown in Fig. 2(c), the $T<T_{N}$ and $T>T_{N} \varepsilon_{a b}$ data are fit to linear functions, and $\Delta \varepsilon_{a b}$ is determined to be the difference between these two fittings at $T_{N}$. The $\Delta \varepsilon_{a b}$ and $\delta$ (based on the data reported in Ref. [19]) values are listed in Table I. In Fig. 5, we plot $\left|J_{1 z}-J_{2 z}\right|$ vs $\delta$ and $\Delta \varepsilon_{a b}$ vs $\delta$ for $\mathrm{YMnO}_{3}, \mathrm{Y}_{0.7} \mathrm{Lu}_{0.3} \mathrm{MnO}_{3}$, and $\mathrm{LuMnO}_{3}$. It shows that both $\left|J_{1 z}-J_{2 z}\right|$ and $\Delta \varepsilon_{a b}$ decrease with decreasing $\delta$, indicating strong correlations between the

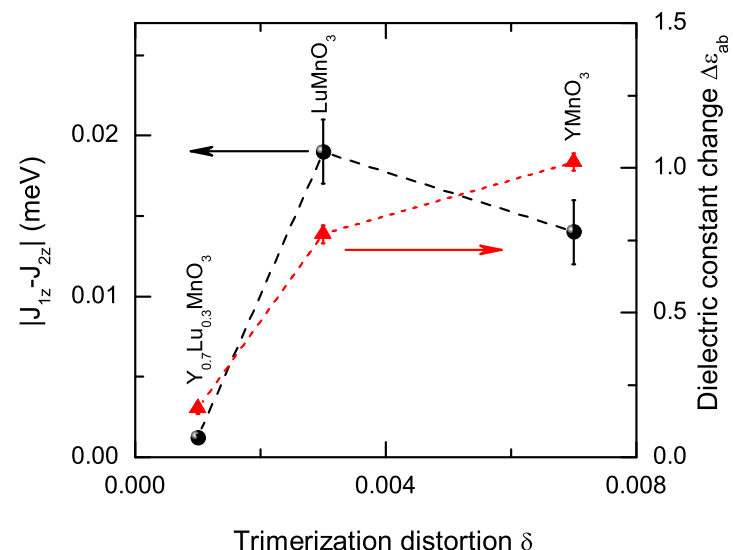

FIG. 5. (Color online) $\left|J_{1 z}-J_{2 z}\right|$ versus trimerization distortion $\delta$ (circle, left axis), and critical dielectric constant change $\Delta \varepsilon_{a b}$ versus $\delta$ (triangle, right axis) for $\mathrm{Y}_{0.7} \mathrm{Lu}_{0.3} \mathrm{MnO}_{3}, \mathrm{YMnO}_{3}$, and $\mathrm{LuMnO}_{3}$.

strength of trimerization distortion and the magnitude of $\mathrm{ME}$ coupling.

In summary, our INS study reveals a smaller $\Delta_{12}$ gap that coincides with a weaker $\varepsilon_{a b}$ dielectric anomaly in $\mathrm{Y}_{0.7} \mathrm{Lu}_{0.3} \mathrm{MnO}_{3}$. This is attributed to a much weaker $\mathrm{Mn}$ trimerization distortion due to the doping influence: the $\mathrm{Mn}$ atomic position $x$ in $\mathrm{Y}_{0.7} \mathrm{Lu}_{0.7} \mathrm{MnO}_{3}$ is very close to $x_{c}=1 / 3$. These results provide strong evidence that the magnitude of ME coupling is linked to the strength of the trimerization distortion, suggesting the $\mathrm{Mn}$ trimerization is responsible for the $\mathrm{ME}$ effect in $\mathrm{Y}_{1-y} \mathrm{Lu}_{y} \mathrm{MnO}_{3}$. Since a high-resolution neutron diffraction study has shown that the Mn trimerization is a systematic feature in $\mathrm{RMnO}_{3}$ [26], our finding may shed light on a deeper understanding of the multiferroic phenomenon in this series of materials, inviting further theoretical investigations.

We acknowledge valuable discussions with Randy Fishman. Work at the High Flux Isotope Reactor and Spallation Neutron Source, Oak Ridge National Laboratory, was sponsored by the Scientific User Facilities Division, Office of Basic Energy Sciences, U.S. Department of Energy. The single crystal growth and neutron scattering work at UTK/Rice is supported by the US NSF Grants No. DMR-1308603 and No. OISE-0968226. The work at Beijing Normal University was supported by Beijing Natural Science Foundation under Contract No. 2132023; J.Z. also acknowledges the Fundamental Research Funds for the Central Universities (2012LYB07).
[1] M. Fiebig, J. Phys. D: Appl. Phys. 38, R123 (2005).

[2] N. A. Spaldin and M. Fiebig, Science 309, 391 (2005).

[3] Y. Tokura, Science 312, 1481 (2006).

[4] W. Eerenstein, N. D. Mathur, and J. F. Scott, Nature (London) 442, 759 (2006)

[5] T. Kimura, T. Goto, H. Shintani, K. Ishizaka, T. Arima, and Y. Tokura, Nature (London) 426, 55 (2003).

[6] N. Hur, S. Park, P. A. Sharma, J. S. Ahn, S. Guha, and S. W. Cheong, Nature (London) 429, 392 (2004).
[7] S.-W. Cheong and M. Mostovoy, Nat. Mater. 6, 13 (2007).

[8] M. Fiebig, Th. Lottermoser, D. Fröhlich, A. V. Goltsev, and R. V. Pisarev, Nature (London) 419, 818 (2002).

[9] T. Lottermoser, T. Lonkai, U. Amann, D. Hohlwein, J. Ihringer, and M. Fiebig, Nature (London) 430, 541 (2004).

[10] A. B. Harris and G. Lawes, in The Handbook of Magnetism and Advanced Magnetic Materials (Wiley, London, 2006).

[11] T. Choi, Y. Horibe, H. T. Yi, Y. J. Choi, W. Wu, and S.-W. Cheong, Nat. Mater. 9, 253 (2010). 
[12] D. G. Tomuta, S. Ramakrishnan, G. J. Nieuwenhuys, and J. A. Mydosh, J. Phys.: Condens. Matter 13, 4543 (2001).

[13] N. Iwata and K. Kohn, J. Phys. Soc. Jpn. 67, 3318 (1998).

[14] Z. J. Huang, Y. Cao, Y. Y. Sun, Y. Y. Xue, and C. W. Chu, Phys. Rev. B 56, 2623 (1997).

[15] T. Katsufuji, S. Mori, M. Masaki, Y. Moritomo, N. Yamamoto, and H. Takagi, Phys. Rev. B 64, 104419 (2001).

[16] Bas B. Van Aken and Thomas T. M. Palstra, Phys. Rev. B 69, 134113 (2004).

[17] S. Lee, A. Pirogov, M. Kang, K.-H. Jang, M. Yonemura, T. Kamiyama, S.-W. Cheong, F. Gozzo, N. Shin, H. Kimura, Y. Noda, and J.-G. Park, Nature (London) 451, 805 (2008).

[18] I. V. Solovyev, M. V. Valentyuk, and V. V. Mazurenko, Phys. Rev. B 86, 054407 (2012).

[19] J. Park, S. Lee, M. Kang, K.-H. Jang, C. Lee, S. V. Streltsov, V. V. Mazurenko, M. V. Valentyuk, J. E. Medvedeva, T. Kamiyama, and J.-G. Park, Phys. Rev. B 82, 054428 (2010).
[20] T. Chatterji, S. Ghosh, A. Singh, L. P. Regnault, and M. Rheinstädter, Phys. Rev. B 76, 144406 (2007).

[21] O. P. Vajk, M. Kenzelmann, J. W. Lynn, S. B. Kim, and S.-W. Cheong, Phys. Rev. Lett. 94, 087601 (2005).

[22] J. Park, J.-G. Park, G. S. Jeon, H.-Y. Choi, C. Lee, W. Jo, R. Bewley, K. A. McEwen, and T. G. Perring, Phys. Rev. B 68, 104426 (2003).

[23] T. J. Sato, S.-H. Lee, T. Katsufuji, M. Masaki, S. Park, J. R. D. Copley, and H. Takagi, Phys. Rev. B 68, 014432 (2003).

[24] H. J. Lewtas, A. T. Boothroyd, M. Rotter, D. Prabhakaran, H. Müller, M. D. Le, B. Roessli, J. Gavilano, and P. Bourges, Phys. Rev. B 82, 184420 (2010).

[25] M. Rotter et al., MCPHASE, a software package that can calculate the phase diagram and magnetic properties of magnetic materials, 2002-2010, available at http://www.mcphase.de.

[26] X. Fabrèges, S. Petit, I. Mirebeau, S. Pailhès, L. Pinsard, A. Forget, M. T. Fernandez-Diaz, and F. Porcher, Phys. Rev. Lett. 103, 067204 (2009). 